\title{
NOTE ON THE PLÜCKER EQUATIONS FOR PLANE ALGEBRAIC CURVES IN THE GALOIS FIELDS
}

\author{
BY A. D. CAMPBELL
}

In this note we shall consider the Plücker* equations for plane algebraic curves and find out in how far they are valid in the Galois fields of order $p^{n}$. We designate these fields by $G F\left(p^{n}\right)$. Let us consider the $n$-ic

$$
\begin{aligned}
& f(x, y, z) \equiv a z^{n}+\left(b_{0} x+b_{1} y\right) z^{n-1} \\
&+\left(c_{0} x^{2}+c_{1} x y+c_{2} y^{2}\right) z^{n-2} \\
&+\left(d_{0} x^{3}+d_{1} x^{2} y+d_{2} x y^{2}+d_{3} y^{3}\right) z^{n-3}+\cdots \\
&+\left(l_{0} x^{n}+\cdots+l_{n} y^{n}\right)=0,
\end{aligned}
$$

the first polar† of $(0,0,1)$ with respect to (1),

$$
\begin{aligned}
n a z^{n-1} & +(n-1)\left(b_{0} x+b_{1} y\right) z^{n-2} \\
& +(n-2)\left(c_{0} x^{2}+c_{1} x y+c_{2} y^{2}\right) z^{n-3}+\cdots=0,
\end{aligned}
$$

and the polar conic of $(0,0,1)$ with respect to $(1)$,

(3) $\frac{n(n-1)}{2} a z^{2}+(n-1)\left(b_{0} x+b_{1} y\right) z+\left(c_{0} x^{2}+c_{1} x y+c_{2} y^{2}\right)=0$.

In the derivation of the Plücker equations given by Hiltonł, use is made of the number of intersections of the $n$-ic and its first polar and of the $n$-ic and its Hessian, also the polar reciprocal of the $n$-ic with respect to a given conic is utilized. If $n=\alpha p^{r}+1$ the polar conic (3) passes through $(0,0,1)$ and is degenerate, but $(0,0,1)$ can be any point $P$, hence for these $G F\left(p^{n}\right)$ we cannot use the Hessian in the derivation of any of Plücker's equations. Also in the $G F\left(2^{n}\right)$ the polars of all the points in the plane with respect to any given conic

* See Hilton, Plane Algebraic Curves, p. 112.

† See A. D. Campbell, The polar curves of plane algebraic curves in the Galois fields, this Bulletin, vol. 34 (1928), pp. 361-363.

‡ Loc. cit., pp. 97, 100, 63, 34, 66 . 
are concurrent (as is easy to prove), hence in these fields we cannot use the polar reciprocal of the $n$-ic with respect to a conic.

We get around these difficulties in the following manner. Hilton shows* that if we can establish the three following equations, the others follow from these three:

$$
\begin{aligned}
& m=n(n-1)-2 \delta-3 \kappa, \\
& n=m(m-1)-2 \tau-3 \iota, \\
& \text { (6) } \quad \frac{1}{2} n(n+3)-\delta-2 \kappa=\frac{1}{2} m(m+3)-\tau-2 \iota \text {, }
\end{aligned}
$$

where $n$ is the order of the curve under consideration, $m$ is its class, $\delta$ the number of nodes on the curve, $\kappa$ the number of cusps, $\tau$ the number of bitangents, $\iota$ the number of inflections. Just as in Hilton pp. 96 and $97 \dagger$ so here we can readily see that (2) cuts (1) once at the point of contact of each tangent from $(0,0,1)$ to the given curve, twice at each node and thrice at each cusp of the given curve. Also the curves (1) and (2) ordinarily intersect in $n(n-1)$ points, hence equation (4) is in general valid in the $G F\left(p^{n}\right)$. In some cases (1) and (2) do not intersect in $n(n-1)$ points, namely when (2) is composite with a repeated factor. Such a case occurs for $p=2, n=3$, and (1) lacking an $x y z$ term.

The equations (5) and (6) are established in Hilton by taking the polar reciprocal of the $n$-ic with respect to a conic and by the use of Sylvester's dialytic method. $\neq$ The fact is used (p. 63) that from a point on the tangent $t$ at a cusp $P$ only one tangent to the $n$-ic coincides with $t$, while at $P$ three tangents coincide with $t$. This fact is necessary in order to show that the polar reciprocal of a cusp is a point of inflection. The proof of this fact that is given in Hilton (pp. 84, 86) is not applicable to the $G F\left(p^{n}\right)$, so we substitute the following proof. If we take $P$ as $(0,0,1)$ with $y=0$ as tangent,

* Loc. cit., p. 112.

$\dagger$ Using the eliminant as in Hilton, pp. 10, 11.

$\ddagger$ Loc. cit., pp. 97, 63, 66. 
A. D. CAMPBELL [Nov.-Dec.,

then in (1) $a=b_{0}=b_{1}=c_{0}=c_{1}=0$ and $c_{2} \neq 0$, hence the curve* and the first polar of $P$ have in common only $n(n-1)-2 \cdot 2-2$ points beside $P$, whereas for $P^{\prime}$ an ordinary point the curve and the first polar of $P^{\prime}$ have in common $n(n-1)-3$ points beside the cusp $P$. From this follows the above fact. In the $G F\left(p^{n}\right)$ for $p>2$ we can take the polar reciprocal of an $n$-ic with respect to a conic. Also we do not need to use the Hessian because (5) and (6) are proved in Hilton by means of the polar reciprocal of the $n$-ic; $\dagger$ and from (5) and (6) we can obtain what Hilton obtains from the intersections of the $n$-ic and its Hessian, namely the equation $\ddagger$

$$
\iota=3 n(n-2)-6 \delta-8 \kappa .
$$

Therefore the Plücker equations are valid in the $G F\left(p^{n}\right)$ for $p>2$. In the $G F\left(2^{n}\right)$ we cannot use a polar reciprocal of the $n$-ic; we can, however, use plane duality $\S$ in these $G F\left(2^{n}\right)$ to replace the use of the polar reciprocal in proving (5) and (6). Therefore also in the $G F\left(2^{n}\right)$ the Plücker equations are still valid.

Syracuse University

* Hilton, loc. cit., p. 11.

$\dagger$ Loc. cit., pp. 33, 66.

$\ddagger$ Loc. cit., pp. 100, 101.

$\S$ See Veblen and Young, Projective Geometry, vol. 1, p. 201. 